\title{
Analysis and Compensation of DC Offset in OFDM Systems Over Frequency-Selective Rayleigh Fading Channels
}

\author{
Chi-Hsiao Yih
}

\begin{abstract}
Direct-conversion receivers have become popular in radio-frequency ( $R F$ ) circuit design for their advantages of low cost, low power consumption, and fewer chip components over other architectures such as heterodyne receivers. However, the direct-conversion receiver architecture often suffers from direct current (dc) offset, which is a consequence of the imperfect direct-conversion process. In this paper, we study the effects of dc offset on the symbol error rate (SER) performance of orthogonal frequency-division multiplexing (OFDM) systems in multipath Rayleigh fading channels. Since OFDM system performance is sensitive to carrier frequency offset (CFO), the CFO must be estimated and compensated at the receiver. Due to CFO compensation, the dc offset caused by direct-conversion receivers and/or mixed-signal circuits no longer only affects the dc subcarrier and is spread over all subcarriers. By deriving the analytical SER formulas for OFDM systems with various modulation formats, the dependency of SER on dc offset and CFO is clearly quantified. These SER formulas can help system designers determine suitable specifications of RF components and understand whether digital dc offset compensation is necessary or not. Finally, we propose and analyze a simple dc offset estimation and cancellation scheme under the assumption that dc offset holds constant in one OFDM symbol duration. Numerical results demonstrate the effectiveness of the proposed dc offset-cancellation scheme.
\end{abstract}

Index Terms-Carrier frequency offset (CFO), direct current (dc) offset, interference cancellation, orthogonal frequencydivision multiplexing (OFDM), performance analysis, Rayleigh fading, symbol error rate (SER).

\section{INTRODUCTION}

D OWN conversion of radio-frequency (RF) signals to baseband can first be done through an intermediate frequency (IF) and then to baseband. This receiver architecture is called heterodyne receiver. An alternative approach is called directconversion or zero-IF architecture, which directly converts RF signals to baseband without the need for an IF stage. In comparison with heterodyne receivers, direct-conversion receivers have the advantages of low cost, low power consumption, and fewer chip components. However, the direct-conversion receiver architecture often suffers from direct current (dc) offset, which appears as an interference at the center of the desired band. The main mechanisms generating dc offset are local os-

Manuscript received July 22, 2008; revised December 23, 2008. First published February 2, 2009; current version published August 14, 2009. The review of this paper was coordinated by Prof. W. Su.

The author is with the Department of Electrical Engineering, Tamkang University, Tamsui 25137, Taiwan (e-mail: chyih@ee.tku.edu.tw).

Color versions of one or more of the figures in this paper are available online at http://ieeexplore.ieee.org.

Digital Object Identifier 10.1109/TVT.2009.2014240 cillator (LO) leakage self-mixing, LO reradiation self-mixing, and interferer self-mixing [1]. The dc offset is also generated by the analog offset affecting the baseband part of the analog frontend circuits in the transceiver. In addition, transistor mismatch in the signal path and the second-order intermodulation of the RF components, such as low-noise amplifiers, mixers, and analog filters, also produce dc offset.

In a wireless communication environment, the dc offset at the receiver may consist of both static (time-invariant) and dynamic (time-varying) dc offsets. The dc offset generated by transistor mismatches and self-mixing of LO leakage can be considered as the static dc offset. On the other hand, the dc offset generated by self-mixing of reradiated LO leakage and interferer leakage is the dynamic dc offset due to the time variation of wireless channels [2]. The simplest method to remove the static dc offset is to consider high-pass filtering if the dc-free transmission scheme is employed at the transmitter. However, high-pass filters usually demand prohibitively large capacitors and fail to track the dynamic dc offset. Other methods to mitigate the dc offset include subharmonic or superharmonic mixing and offset calibration techniques at the baseband [3].

In addition to the RF front end, the other sources of dc offset are the digital-to-analog converter (DAC) at the transmitter and the analog-to-digital converter (ADC) at the receiver [4]. Without proper compensation, dc offset may cause the receiver to obtain biased estimates of important parameters for signal demodulation and detection. Since the constellation of highorder modulation is denser than that of binary phase shift keying (BPSK) modulation, the performance loss due to dc offset in 64-ary quadrature amplitude modulation (64-QAM) is expected to be much worse than that in BPSK modulation.

The analysis of bit error rate (BER) performance in a direct-conversion receiver with different levels of dc offset for time-division multiple-access (TDMA)-based single-carrier communication systems was investigated in [5]-[7]. In [5], three principal impairments of analog circuits, named gain imbalance, phase imbalance, and dc offset, were considered, and the resulting performance losses in terms of BER and out-of-band power were quantitatively assessed. The author also proposed an adaptive compensation technique to deal with these impairments. When the received signal suffers from fading, a joint channel and dc offset estimation scheme was proposed in [6], which gives competitive receiver performance for the EDGE system. A detailed BER performance analysis for $M$-QAM and $M$-ary phase shift keying ( $M$-PSK) modulations impaired by dc offset in a direct-conversion receiver was 
presented in [7]. Moreover, the authors also devised a simple design rule justifying when the dc offset can be ignored for BPSK modulation.

For multicarrier communication systems, Witrisal [8] analyzed the impact of dc offset and carrier feedthrough on the correlator-based carrier frequency offset (CFO) estimator in an orthogonal frequency-division multiplexing (OFDM) platform. To reduce the performance degradation, the original CFO estimation algorithm given in [9] was modified to cancel the interference caused by dc offset. Another low-complexity frequency offset estimation in the presence of dc offset for OFDM systems based on the concept of sequential estimation was described in [10]. The overall nonidealities, including dc offset, of the directconversion receiver on wireless local area network (WLAN) system performance were simulated and reported in [11] and [12]. A subspace-based CFO estimation scheme for OFDM systems in the presence of dc offset was proposed and analyzed in [13]. Finally, a best linear unbiased estimator (BLUE) for dc offset in OFDM-based WLAN system was derived in [14].

In most practical OFDM systems, the dc subcarrier usually does not transmit any data to avoid the undesirable dc offset introduced in the transceiver. However, it is important to note that the final OFDM baseband signal sent over a channel is not $\mathrm{dc}$ free due to the LO leakage in the mixer and other dc offset sources in the analog front-end circuits of the direct-conversion OFDM transmitter [15], [16]. That means that the dc offset originates from not only the receiver but from the transmitter as well. On the other hand, the CFO between the transmitter and the receiver must be estimated and compensated to maintain good performance [17]. Due to CFO compensation, the energy of dc offset is spread over all subcarriers and no longer only affects the performance of the dc subcarrier. The first objective of this paper is to analytically determine the dependency of symbol error rate (SER) on the CFO and dc offset for OFDM systems in multipath Rayleigh fading channels. We extend our previous work [18] to high-order modulation with both the transmitter and the receiver dc offsets. The second objective of this paper is to design and analyze a dc offset compensation method to mitigate the detrimental effect of dc offset on system performance. Throughout this paper, we focus our study on the static dc offset, which is the major component of dc offset in indoor WLAN applications.

The remainder of this paper is organized as follows. In Section II, we describe the system model. The SER analysis of OFDM systems impaired by dc offset and residual CFO is presented in Section III. A simple dc offset estimation and cancellation scheme is proposed, and its performance is analyzed in Section IV. Numerical results are given in Section V, and conclusions are drawn in Section VI.

\section{SySTEM MODEL}

The discrete baseband OFDM signal transmitted in one OFDM symbol period can be implemented by the inverse discrete Fourier transform (IDFT) as

$x_{n}=\frac{1}{\sqrt{N}} \sum_{m \in \mathcal{I}} X_{m} e^{j 2 \pi n m / N}+\eta_{t}, \quad n=0,1, \ldots, N-1$ where $N$ is the number of subcarriers in an OFDM system, $m$ is the index of subcarrier, $\mathcal{I}$ is a subset of the set $\{-N / 2,-N / 2+$ $1, \ldots, N / 2-1\}$, and $X_{m} \in \mathcal{X}$ is the transmitted modulation symbol at the $m$ th subcarrier. The modulation symbols $X_{m}$ 's are assumed to be independent. Three types of constellations $\mathcal{X}$, namely, BPSK, QPSK, and 16-QAM, are considered in this paper. The additive term $\eta_{t}$ represents the static dc offset generated by the direct-conversion OFDM transmitter.

The elements of the active set $\mathcal{I}$ represent the indices of subcarriers used for data transmission. We assume 0 does not belong to the active set $\mathcal{I}$, which means that there is no information transmitted at the dc subcarrier. The dc-free transmission scheme is typical in most practical OFDM-based communication systems (e.g., 802.11a [19] and digital video broadcasting-terrestrial (DVB-T) [20]) so that the dc offset resulting from the direct-conversion receiver can easily be filtered out without affecting the transmitted data symbols. Since the number of subcarriers in an OFDM system is usually large, the throughput loss due to dc-free transmission is almost negligible.

To eliminate intersymbol interference (ISI), we assume that the duration of cyclic prefix is longer than the maximum delay spread of a multipath fading channel. After the insertion of a cyclic prefix, the OFDM signal passes through a quasi-static multipath Rayleigh fading channel whose impulsive response is given by

$$
h(t)=\sum_{l=0}^{L-1} h_{l} \delta(t-l T / N)
$$

where $L$ is the number of multipaths, the path gains $h_{l}$ are independent complex Gaussian random variables with mean 0 and variance $\sigma_{l}^{2}, \delta(\cdot)$ is the Dirac-delta function, and $T$ is the effective OFDM symbol period. We assume that the path gains $h_{l}$ hold constant in one frame and independently change from one frame to another. The channel frequency response at subcarrier $i$ is

$$
H_{i}=\int_{-\infty}^{\infty} h(t) e^{-2 \pi i t / T} d t=\sum_{l=0}^{L-1} h_{l} e^{-j 2 \pi i l / N} .
$$

Since $h_{l}$ 's are independent complex Gaussian random variables, $H_{i}$ 's are correlated complex Gaussian random variables with mean 0 and variance $\sum_{l=0}^{L-1} \sigma_{l}^{2}$. The correlation between $H_{i}$ and $H_{k}$ is

$$
\rho_{i k}=\mathbb{E}\left[H_{i} H_{k}^{*}\right]=\sum_{l=0}^{L-1} \sigma_{l}^{2} e^{j 2 \pi(k-i) l / N}
$$

where $\mathbb{E}[\cdot]$ denotes statistical expectation, and $x^{*}$ represents the complex conjugation of the complex number $x$. Without loss of generality, we assume that the variance of $H_{i}$ is normalized to 1, i.e., $\sum_{l=0}^{L-1} \sigma_{l}^{2}=1$.

Let $f_{\Delta}$ be the CFO between the transmitter and the receiver, and let $\eta_{r}$ be the complex-valued dc offset generated by the receiver. Moreover, we assume that $\eta_{r}$ is time invariant over 
one OFDM symbol period. Then, the received OFDM signal after cyclic prefix removal is given by

$$
\begin{array}{r}
y_{n}=\frac{1}{\sqrt{N}} \sum_{m \in \mathcal{I}} H_{m} X_{m} e^{j 2 \pi n(m+\epsilon) / N}+H_{0} \eta_{t} e^{j 2 \pi n \epsilon / N}+\eta_{r}+w_{n} \\
n=0,1, \ldots, N-1
\end{array}
$$

where $\epsilon=f_{\Delta} T$ is the normalized CFO, and $w_{n}$ 's are the additive noises modeled by independently circularly symmetric complex Gaussian random variables with mean 0 and variance $N_{0} / 2$ in real and imaginary components.

Let $\hat{\epsilon}$ be the estimated normalized CFO. Then, the received signal $y_{n}$ after CFO compensation and discrete Fourier transform (DFT) is given by

$$
\begin{aligned}
Y_{k} & =\frac{1}{\sqrt{N}} \sum_{n=0}^{N-1} y_{n} e^{-j 2 \pi n(k+\hat{\epsilon}) / N} \\
k & =-N / 2,-N / 2+1, \ldots, N / 2-1 .
\end{aligned}
$$

After substituting (5) into (6) and performing some straightforward algebra, we have

$$
Y_{k}=\alpha H_{k} X_{k}+I_{k}^{\mathrm{ICI}}+I_{k}^{\mathrm{DC}}+W_{k}
$$

where $\alpha=e^{j \pi(\epsilon-\hat{\epsilon})((N-1) / N)}(\operatorname{sinc}(\epsilon-\hat{\epsilon}) / \operatorname{sinc}((\epsilon-\hat{\epsilon}) / N)), \operatorname{sinc}(x)=$ $(\sin (\pi x) / \pi x), I_{k}^{\mathrm{ICI}}=\sum_{m \in \mathcal{I}, m \neq k} H_{m} X_{m} e^{j \pi(m-k+\epsilon-\hat{\epsilon})((N-1) / N)}$ $(\operatorname{sinc}(m-k+\epsilon-\hat{\epsilon}) / \operatorname{sinc}((m-k+\epsilon-\hat{\epsilon}) / N))$, and $I_{k}^{\mathrm{DC}}=$ $H_{0} \eta_{t} \sqrt{N} e^{-j \pi(k+\hat{\epsilon} \epsilon)((N-1) / N)}(\operatorname{sinc}(k+\hat{\epsilon}-\epsilon) / \operatorname{sinc}((k+\hat{\epsilon}-\epsilon) / N))+$ $\eta_{r} \sqrt{N} e^{-j \pi(k+\hat{\epsilon})((N-1) / N)}(\operatorname{sinc}(k+\hat{\epsilon}) / \operatorname{sinc}((k+\hat{\epsilon}) / N))$. The additive noise terms $W_{k}=(1 / \sqrt{N}) \sum_{n=0}^{N-1} w_{n} e^{-j 2 \pi(k+\hat{\epsilon}) n / N}$ are still independently circularly symmetric complex Gaussian random variables with mean 0 and variance $N_{0} / 2$ in both real and imaginary parts. We consider the following two special cases to illustrate the interaction between dc offset interference and $\mathrm{CFO}$ correction.

Case 1-Without CFO Correction $\hat{\epsilon}=0$ : This means that the normalized CFO $\epsilon$ is not compensated. In this case, the effects of dc offset and CFO on the received OFDM signals are characterized by

$$
\begin{aligned}
& I_{k}^{\mathrm{DC}} \\
& =H_{0} \eta_{t} \sqrt{N} e^{-j \pi(k-\epsilon) \frac{N-1}{N}} \frac{\operatorname{sinc}(k-\epsilon)}{\operatorname{sinc}\left(\frac{k-\epsilon}{N}\right)} \\
& \quad+\eta_{r} \sqrt{N} e^{-j \pi k \frac{N-1}{N}} \frac{\operatorname{sinc}(k)}{\operatorname{sinc}(k / N)} \\
& =\left\{\begin{array}{l}
H_{0} \eta_{t} \sqrt{N} e^{j \pi \epsilon \frac{N-1}{N}} \frac{\operatorname{sinc}(\epsilon)}{\operatorname{sinc}(\epsilon / N)}+\eta_{r} \sqrt{N}, \quad \text { when } k=0 \\
H_{0} \eta_{t} \sqrt{N} e^{-j \pi(k-\epsilon) \frac{N-1}{N}} \frac{\operatorname{sinc}(k-\epsilon)}{\operatorname{sinc}\left(\frac{k-\epsilon}{N}\right)},
\end{array}\right. \\
& I_{k}^{\mathrm{ICI}} \quad \text { otherwise } \\
& =\sum_{m \in \mathcal{I}, m \neq k} H_{m} X_{m} e^{j \pi(m-k+\epsilon) \frac{N-1}{N}} \frac{\operatorname{sinc}(m-k+\epsilon)}{\operatorname{sinc}\left(\frac{m-k+\epsilon}{N}\right)} .
\end{aligned}
$$

Since the dc subcarrier does not transmit any information, the dc offset $\eta_{r}$ has no effect on system performance. On the other hand, the CFO spreads the energy of dc offset $\eta_{t}$ over all subcarriers. Finally, the lack of CFO compensation results in serious performance degradation due to the intercarrier interference (ICI) caused by the CFO.

Case 2-Perfect CFO Correction $\hat{\epsilon}=\epsilon$ : When the CFO is perfectly compensated, we have

$$
\begin{aligned}
I_{k}^{\mathrm{DC}}= & H_{0} \eta_{t} 0 \sqrt{N} e^{-j \pi k \frac{N-1}{N}} \frac{\operatorname{sinc}(k)}{\operatorname{sinc}(k / N)} \\
& +\eta_{r} \sqrt{N} e^{-j \pi(k+\epsilon) \frac{N-1}{N}} \frac{\operatorname{sinc}(k+\epsilon)}{\operatorname{sinc}\left(\frac{k+\epsilon}{N}\right)} \\
& = \begin{cases}H_{0} \eta_{t} \sqrt{N}+\eta_{r} \sqrt{N} e^{-j \pi \epsilon \frac{N-1}{N}} \frac{\operatorname{sinc}(\epsilon)}{\operatorname{sinc}(\epsilon / N)}, & \text { when } k=0 \\
\eta_{r} \sqrt{N} e^{-j \pi(k+\epsilon) \frac{N-1}{N}} \frac{\operatorname{sinc}(k+\epsilon)}{\operatorname{sinc}\left(\frac{k+\epsilon}{N}\right)}, & \text { otherwise }\end{cases} \\
I_{k}^{\mathrm{ICI}}= & 0 .
\end{aligned}
$$

In this case, the dc offset $\eta_{t}$ only appears at the dc subcarrier. For all subcarriers except the dc subcarrier, the interference is completely contributed from the receiver dc offset $\eta_{r}$ whose energy is spread over all subcarriers by the CFO compensation. Therefore, the interferences $I_{k}^{\mathrm{DC}}$ and $I_{k}^{\mathrm{ICI}}$ depend on how perfectly the $\mathrm{CFO}$ compensation is done.

In what follows, we analyze the joint effects of dc offset and residual CFO on the SER of OFDM systems in multipath Rayleigh fading channels. As for the CFO estimation algorithm in the presence of carrier feedthrough and dc offset, see [8] and [21].

\section{BIT ERROR RATE ANALYSIS}

The constellation of BPSK modulation is denoted by $\mathcal{X}=\left\{\sqrt{E_{s}},-\sqrt{E_{s}}\right\}$, where $E_{s}$ represents the average symbol energy. We assume that the two constellation points are equally likely to be chosen for transmission. For subcarrier $k$, the SER under the assumption of perfect channel state information (CSI) at the receiver is

$$
\begin{aligned}
P_{s}^{\mathrm{BPSK}}(k)=\frac{1}{2} & {\left[\operatorname{Pr}\left(\operatorname{Re}\left[Y_{k} H_{k}^{*}\right]<0 \mid X_{k}=\sqrt{E_{s}}\right)\right.} \\
& \left.+\operatorname{Pr}\left(\operatorname{Re}\left[Y_{k} H_{k}^{*}\right]>0 \mid X_{k}=-\sqrt{E_{s}}\right)\right]
\end{aligned}
$$

where the notation $\operatorname{Re}[x]$ denotes the real part of the complex number $x$. It is worth mentioning that the two probabilities on the right-hand side (RHS) of (12) may not be the same due to the presence of the transmitter dc offset $\eta_{t}$.

Since the channel frequency responses $H_{k}$ are correlated, the signal power and the interference power are correlated. Directly applying the central limit theorem (CLT) to approximate the ICI term as a Gaussian random variable does not predict the true SER well, since it ignores the correlation between the signal power and the interference power [17]. To perform accurate 
SER analysis, we adopt the approach presented in [17]. First, we compute the conditional SER as

$$
\begin{aligned}
P_{s}^{\operatorname{BPSK}}\left(k \mid H_{k}\right) & \\
=\frac{1}{2}[ & \operatorname{Pr}\left(\operatorname{Re}\left[Y_{k} H_{k}^{*}\right]<0 \mid X_{k}=\sqrt{E_{s}}, H_{k}\right) \\
& \left.\quad+\operatorname{Pr}\left(\operatorname{Re}\left[Y_{k} H_{k}^{*}\right]>0 \mid X_{k}=-\sqrt{E_{s}}, H_{k}\right)\right] .
\end{aligned}
$$

Then, we find the unconditional SER by taking the expectation of $P_{s}^{\mathrm{BPSK}}\left(k \mid H_{k}\right)$ with respect to the probability density function (pdf) of $H_{k}$. For $k \in \mathcal{I}$, the decision variable $Y_{k} H_{k}^{*}$ conditioned on $X_{k}$ and $H_{k}$ is given by

$$
\begin{aligned}
& Y_{k} H_{k}^{*}=\alpha\left|H_{k}\right|^{2} X_{k} \\
&+\left[\sum_{m \in \mathcal{I}, m \neq k} \Lambda_{m k} X_{m} \psi(m-k, \epsilon-\hat{\epsilon})\right. \\
&+\Lambda_{0 k} \eta_{t} \sqrt{N} \psi(-k, \epsilon-\hat{\epsilon}) \\
&\left.+\eta_{r} \sqrt{N} \psi(-k,-\hat{\epsilon})+W_{k}\right] H_{k}^{*}
\end{aligned}
$$

where

$$
\psi(a, b)=e^{j \pi(a+b) \frac{N-1}{N}} \frac{\operatorname{sinc}(a+b)}{\operatorname{sinc}\left(\frac{a+b}{N}\right)} .
$$

The conditional random variable $H_{m} \mid H_{k}$ is denoted by $\Lambda_{m k}$, which is also a complex Gaussian random variable with mean $\rho_{m k} H_{k}$ and variance $1-\left|\rho_{m k}\right|^{2}$. To facilitate later analysis, we define a zero-mean complex Gaussian random variable $\Omega_{m k}=$ $\Lambda_{m k}-\rho_{m k} H_{k}$.

Substituting $\Lambda_{m k}=\Omega_{m k}+\rho_{m k} H_{k}$ into (14) and rearranging the equation, we have

$$
\begin{aligned}
Y_{k} H_{k}^{*}=\alpha\left|H_{k}\right|^{2} X_{k}+ & {\left[\left(\Omega_{0 k}+\rho_{0 k} H_{k}\right) \eta_{t} \sqrt{N} \psi(-k, \epsilon-\hat{\epsilon})\right.} \\
& \left.+\eta_{r} \sqrt{N} \psi(-k,-\hat{\epsilon})+W_{k}\right] H_{k}^{*} \\
+ & \sum_{\begin{array}{c}
m \in \mathcal{I}, m \neq k \\
\end{array}}\left(\Omega_{m k}+\rho_{m k} H_{k}\right) \\
& \times X_{m} \psi(m-k, \epsilon-\hat{\epsilon}) H_{k}^{*} .
\end{aligned}
$$

The last term in (16) is the ICI term, which consists of two parts. Define two new random variables as

$$
\begin{aligned}
U_{k} & =\sum_{m \in \mathcal{I}, m \neq k} \Omega_{m k} X_{m} \psi(m-k, \epsilon-\hat{\epsilon}) \\
V_{k} & =\sum_{m \in \mathcal{I}, m \neq k} \rho_{m k} X_{m} \psi(m-k, \epsilon-\hat{\epsilon}) .
\end{aligned}
$$

When the cardinality of the set $\mathcal{I}$ is reasonably large, we can evoke the CLT to approximate $U_{k}$ and $V_{k}$ as complex Gaussian random variables with mean zero and variances $\sigma_{U_{k}}^{2}=\sum_{m \in \mathcal{I}, m \neq k}\left(1-\left|\rho_{m k}\right|^{2}\right) E_{s}|\psi(m-k, \epsilon-\hat{\epsilon})|^{2}$ and $\sigma_{V_{k}}^{2}=$ $\sum_{m \in \mathcal{I}, m \neq k}\left|\rho_{m k}\right|^{2} E_{s}|\psi(m-k, \epsilon-\hat{\epsilon})|^{2}$, respectively. After conditioning on $H_{k}$ and approximating the conditional ICI terms as Gaussian random variables, we rewrite (16) as

$$
Y_{k} H_{k}^{*}=\alpha\left|H_{k}\right|^{2} X_{k}+Z_{k}
$$

where

$$
\begin{aligned}
Z_{k}=\left[U_{k}+H_{k} V_{k}+\left(\Omega_{0 k}+\right.\right. & \left.\rho_{0 k} H_{k}\right) \eta_{t} \sqrt{N} \psi(-k, \epsilon-\hat{\epsilon}) \\
& \left.+\eta_{r} \sqrt{N} \psi(-k,-\hat{\epsilon})+W_{k}\right] H_{k}^{*}
\end{aligned}
$$

is a complex Gaussian random variable with mean $\mu_{z_{k}}$ and variance $\sigma_{z_{k}}^{2}$, i.e.,

$$
\begin{aligned}
& \mu_{z_{k}}= \sqrt{N}\left[\rho_{0 k} H_{k} \eta_{t} \psi(-k, \epsilon-\hat{\epsilon})+\eta_{r} \psi(-k,-\hat{\epsilon})\right] H_{k}^{*} \\
& \sigma_{z_{k}}^{2}=\left[\sigma_{U_{k}}^{2}+\left|H_{k}\right|^{2} \sigma_{V_{k}}^{2}+\left(1-\left|\rho_{0 k}\right|^{2}\right)\left|\eta_{t}\right|^{2}\right. \\
&\left.\quad \times N|\psi(-k, \epsilon-\hat{\epsilon})|^{2}+N_{0}\right]\left|H_{k}\right|^{2} .
\end{aligned}
$$

From the mean of $Z_{k}$, we know the dc offsets $\eta_{t}$ and $\eta_{r}$ cause the constellation to deviate from its original location. On the other hand, only the transmitter dc offset $\eta_{t}$ increases the interference variance. The increment of interference variance due to the transmitter dc offset $\eta_{t}$ also depends on the correlation of the channel frequency responses through $1-\left|\rho_{0 k}\right|^{2}$ and the CFO through $|\psi(-k, \epsilon-\hat{\epsilon})|^{2}$.

The conditional SER [see (13)] can be expressed as

$$
\begin{aligned}
P_{s}^{\operatorname{BPSK}}\left(k \mid H_{k}\right)=\frac{1}{2} & {\left[Q\left(\frac{\operatorname{Re}\left[\alpha\left|H_{k}\right|^{2} \sqrt{E_{s}}+\mu_{z_{k}}\right]}{\sqrt{\sigma_{z_{k}}^{2} / 2}}\right)\right.} \\
& \left.+Q\left(\frac{\operatorname{Re}\left[\alpha\left|H_{k}\right|^{2} \sqrt{E_{s}}-\mu_{z_{k}}\right]}{\sqrt{\sigma_{z_{k}}^{2} / 2}}\right)\right]
\end{aligned}
$$

where $Q(x)=(1 / \sqrt{2 \pi}) \int_{x}^{\infty} e^{-t^{2} / 2} d t$. Finally, the unconditional SER is

$$
P_{s}^{\mathrm{BPSK}}(k)=\mathbb{E}_{H_{k}}\left[P_{s}^{\mathrm{BPSK}}\left(k \mid H_{k}\right)\right]
$$

where $\mathbb{E}_{H_{k}}[\cdot]$ means that the expectation is taken with respect to the pdf of $H_{k}$. Since $H_{k}$ is a zero-mean unit-variance complex Gaussian random variable, the computation of $P_{s}^{\mathrm{BPSK}}(k)$ involves a double integral. The double integral can accurately be evaluated by using the Gauss-Chebyshev quadrature rule [22], which has been implemented in most numerical computation packages.

From (20), we know that only the magnitude of $\eta_{r}$ affects $P_{s}^{\mathrm{BPSK}}(k)$, and the phase of $\eta_{r}$ is irrelevant to the value of $P_{s}^{\operatorname{BPSK}}(k)$ because the fading gains $H_{k}$ are all circularly symmetric Gaussian random variables. However, $P_{s}^{\mathrm{BPSK}}(k)$ depends on both the phase and the magnitude of $\eta_{t}$. That is the reason why we need to compute the SER for each constellation point. Since $\mu_{z_{k}}$ and $\sigma_{z_{k}}^{2}$ are functions of the subcarrier index $k$, the effect of dc offset on the SER of each subcarrier is different. When the normalized CFO $\epsilon$ is less than 0.5 , the dc subcarrier 


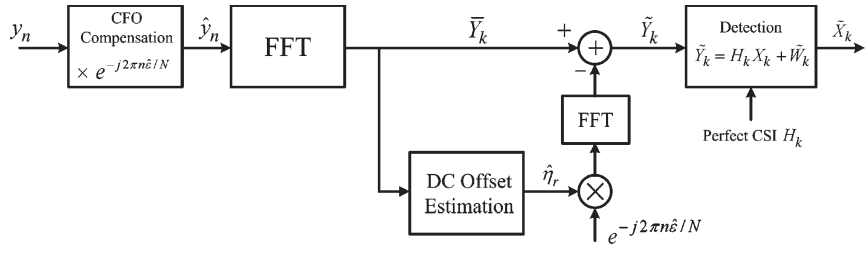

Fig. 1. Block diagram of dc offset estimation and cancellation.

most significantly suffers from the dc offset, and the performance loss decreases as the magnitude of the subcarrier index $|k|$ increases. Finally, the average SER over all subcarriers in the active set $\mathcal{I}$ is

$$
P_{s}^{\mathrm{BPSK}}=\frac{1}{|\mathcal{I}|} \sum_{k \in \mathcal{I}} P_{s}^{\mathrm{BPSK}}(k)
$$

where $|\mathcal{I}|$ is the cardinality of the set $\mathcal{I}$.

Before leaving this section, we have the following two remarks. First, the dc offsets $\eta_{t}$ and $\eta_{r}$ are modeled as complex numbers in this paper. If the dc offsets $\eta_{t}$ and $\eta_{r}$ are represented by random variables with pdfs $p\left(\eta_{t}\right)$ and $p\left(\eta_{r}\right)$, then our previous analytical results can be generalized in this case by taking the expectation of the derived average SER formulas with respect to the pdfs $p\left(\eta_{t}\right)$ and $p\left(\eta_{r}\right)$, respectively. Second, the foregoing BER analysis can be extended to QPSK and 16-QAM modulations, the details of which can be found in the Appendix.

\section{DC OfFSET CANCELLATION}

\section{A. DC Offset Estimation and Cancellation}

In this section, we design and analyze a novel dc offset estimation and cancellation scheme to mitigate the effect of dc offset on the performance of OFDM systems. Since most practical OFDM systems must keep the residual CFO small for good performance, the total degradation caused by dc offset is dominated by the receiver dc offset $\eta_{r}$. Therefore, we solely pay our attention on how to effectively estimate and correct $\eta_{r}$.

The block diagram of the proposed dc offset estimation and cancellation scheme is shown in Fig. 1. The basic idea behind the proposed scheme is that the dc offset $\eta_{r}$ has most energy around the dc subcarrier, and we should use the received signals at subcarriers around the dc subcarrier for the estimation of $\eta_{r}$. For subcarriers far from the dc subcarrier, the energy of dc offset $\eta_{r}$ is too small to yield a reliable estimate. To facilitate $\eta_{r}$ estimation, we assume that the subcarriers $-1,0$, and 1 are all null subcarriers, which implies that $X_{-1}, X_{0}$, and $X_{-1}$ are all equal to zero, and the subcarrier indices $-1,0$, and 1 are not in the active set $\mathcal{I}$. Compared with most existing OFDM-based standards, our proposed scheme has two extra unmodulated subcarriers -1 and 1 . When the number of subcarriers $N$ is large, the loss of spectral efficiency due to the unmodulated subcarriers is negligible.

The received OFDM signal after CFO compensation and DFT has been given in (7). Since the data symbols of the subcarriers $X_{-1}, X_{0}$, and $X_{1}$ are equal to 0 , the received signals at subcarriers $-1,0$, and 1 are given by

$$
\begin{aligned}
Y_{-1}= & I_{-1}^{\mathrm{ICI}}+H_{0} \eta_{t} \sqrt{N} \psi(1, \epsilon-\hat{\epsilon}) \\
& +\eta_{r} \sqrt{N} \psi(1,-\hat{\epsilon})+W_{-1} \\
Y_{0}= & I_{0}^{\mathrm{ICI}}+H_{0} \eta_{t} \sqrt{N} \psi(0, \epsilon-\hat{\epsilon}) \\
& +\eta_{r} \sqrt{N} \psi(0,-\hat{\epsilon})+W_{0} \\
Y_{1}= & I_{1}^{\mathrm{ICI}}+H_{0} \eta_{t} \sqrt{N} \psi(-1, \epsilon-\hat{\epsilon}) \\
& +\eta_{r} \sqrt{N} \psi(-1,-\hat{\epsilon})+W_{1} .
\end{aligned}
$$

Because the receiver does not have knowledge of the true CFO $\epsilon$, the dc offset estimator always assumes $\epsilon$ to be equal to $\hat{\epsilon}$, and the mismatch between $\epsilon$ and $\hat{\epsilon}$ contributes to the estimation error. When $\epsilon=\hat{\epsilon}$, (25)-(27) reduce to

$$
\begin{aligned}
Y_{-1} & =\eta_{r} \sqrt{N} \psi(1,-\hat{\epsilon})+W_{-1} \\
Y_{0} & =H_{0} \eta_{t} \sqrt{N}+\eta_{r} \sqrt{N} \psi(0,-\hat{\epsilon})+W_{0} \\
Y_{1} & =\eta_{r} \sqrt{N} \psi(-1,-\hat{\epsilon})+W_{1} .
\end{aligned}
$$

From (28)-(30), we know the received signal $Y_{0}$ has the largest energy of $\eta_{r}$ among $Y_{-1}, Y_{0}$, and $Y_{1}$ when $|\hat{\epsilon}|<0.5$. In general, it is difficult to estimate $\eta_{r}$ based on $Y_{0}$ because it also contains the unknown transmitter dc offset $\eta_{t}$. However, there is one exception. When $\hat{\epsilon}=0$, we can employ $Y_{0} / \sqrt{N}$ to estimate the total dc offset $H_{0} \eta_{t}+\eta_{r}$ since the total dc offset in $y_{n}$ is exactly equal to $H_{0} \eta_{t}+\eta_{r}$ when $\epsilon=0$.

When $\hat{\epsilon} \neq 0$, we cannot estimate the dc offset $\eta_{r}$ from $Y_{0}$ alone. To suppress the effect of $\eta_{t}$, we form the maximumlikelihood estimate of $\eta_{r}$ based on (28) and (30) as

$$
\hat{\eta}_{r}=\frac{Y_{-1} \psi^{*}(1,-\hat{\epsilon})+Y_{1} \psi^{*}(-1,-\hat{\epsilon})}{\sqrt{N}\left(|\psi(1,-\hat{\epsilon})|^{2}+|\psi(-1,-\hat{\epsilon})|^{2}\right)}, \quad \hat{\epsilon} \neq 0 .
$$

To determine the estimation error, we substitute (25) and (27) into (31). The dc offset estimation error $\Delta \eta_{r}=\hat{\eta}_{r}-\eta_{r}$ is given in (32), shown at the bottom of the next page, where

$\zeta_{1}(\epsilon, \hat{\epsilon})=\sqrt{N}\left[\psi^{*}(1,-\hat{\epsilon}) \psi(1, \epsilon-\hat{\epsilon})+\psi^{*}(-1,-\hat{\epsilon}) \psi(-1, \epsilon-\hat{\epsilon})\right]$.

It is obvious that the dc offset estimate $\hat{\eta}_{r}$ is an unbiased estimator of $\eta_{r}$. Since the mean of $\Delta \eta_{r}$ is zero, the variance of $\Delta \eta_{r}$ is given in (33), shown at the bottom of the next page, where $\zeta_{2}(\epsilon, \hat{\epsilon})$ is expressed as (34), shown at the bottom of the next page.

Once the dc offset estimate $\hat{\eta}_{r}$ is available, we can cancel the dc offset $\eta_{r}$ from the received signal $y_{n}$ and then correct the $\mathrm{CFO}$ as

$$
\tilde{y}_{n}=\left(y_{n}-\hat{\eta}_{r}\right) e^{-j 2 \pi n \hat{\epsilon} / N} .
$$

Taking DFT of the CFO and dc offset corrected signals $\tilde{y}_{n}$, $n=0,1, \ldots, N-1$, we have

$$
\tilde{Y}_{k}=\alpha H_{k} X_{k}+I_{k}^{\mathrm{ICI}}+\tilde{I}_{k}^{\mathrm{DC}}+W_{k}, \quad k \in \mathcal{I}
$$

where

$$
\tilde{I}_{k}^{\mathrm{DC}}=H_{0} \eta_{t} \sqrt{N} \psi(-k, \epsilon-\hat{\epsilon})-\Delta \eta_{r} \sqrt{N} \psi(-k,-\hat{\epsilon}) .
$$


Substituting (32) and (37) into (36), we have

$$
\begin{aligned}
\widetilde{Y}_{k}= & \alpha H_{k} X_{k}+I_{k}^{\mathrm{ICI}}+\xi_{1}(k, \hat{\epsilon}) I_{-1}^{\mathrm{ICI}} \\
& +\xi_{2}(k, \hat{\epsilon}) I_{1}^{\mathrm{ICI}}+H_{0} \eta_{t} \xi_{3}(k, \epsilon, \hat{\epsilon}) \\
& +\xi_{1}(k, \hat{\epsilon}) W_{-1}+\xi_{2}(k, \hat{\epsilon}) W_{1}+W_{k}
\end{aligned}
$$

where

$$
\begin{aligned}
& \xi_{1}(k, \hat{\epsilon})=-\frac{\psi(-k,-\hat{\epsilon}) \psi^{*}(1,-\hat{\epsilon})}{|\psi(1,-\hat{\epsilon})|^{2}+|\psi(-1,-\hat{\epsilon})|^{2}} \\
& \xi_{2}(k, \hat{\epsilon})=-\frac{\psi(-k,-\hat{\epsilon}) \psi^{*}(-1,-\hat{\epsilon})}{|\psi(1,-\hat{\epsilon})|^{2}+|\psi(-1,-\hat{\epsilon})|^{2}} \\
& \xi_{3}(k, \epsilon, \hat{\epsilon})=\sqrt{N} \psi(-k, \epsilon-\hat{\epsilon}) \\
&-\frac{\zeta_{1}(\epsilon, \hat{\epsilon}) \psi(-k,-\hat{\epsilon})}{|\psi(1,-\hat{\epsilon})|^{2}+|\psi(-1,-\hat{\epsilon})|^{2}} .
\end{aligned}
$$

\section{B. BER Analysis}

To evaluate the BER performance after dc offset cancellation, the approach adopted in the previous section is still applicable. We focus on the case $\hat{\epsilon} \neq 0$. For $k \in \mathcal{I}$, the decision variable $\widetilde{Y}_{k} H_{k}^{*}$ conditioned on $X_{k}$ and $H_{k}$ is

$$
\begin{aligned}
& \tilde{Y}_{k} H_{k}^{*}=\alpha\left|H_{k}\right|^{2} X_{k} \\
&+\left[\sum_{m \in \mathcal{I}, m \neq k} \Lambda_{m k} X_{m} \psi(m-k, \epsilon-\hat{\epsilon})\right. \\
&+\xi_{1}(k, \hat{\epsilon}) \sum_{m \in \mathcal{I}} \Lambda_{m k} X_{m} \psi(m+1, \epsilon-\hat{\epsilon}) \\
&+\xi_{2}(k, \hat{\epsilon}) \sum_{m \in \mathcal{I}} \Lambda_{m k} X_{m} \psi(m-1, \epsilon-\hat{\epsilon}) \\
&+\Lambda_{0 k} \eta_{t} \xi_{3}(k, \epsilon, \hat{\epsilon})+\xi_{1}(k, \hat{\epsilon}) W_{-1} \\
&\left.+\xi_{2}(k, \hat{\epsilon}) W_{1}+W_{k}\right] H_{k}^{*} .
\end{aligned}
$$

Substituting $\Lambda_{m k}=\Omega_{m k}+\rho_{m k} H_{k}$ into (42) and rearranging the equation, we have

$$
\begin{aligned}
\widetilde{Y}_{k} H_{k}^{*}=\alpha\left|H_{k}\right|^{2} X_{k} \\
+\left[\hat{U}_{k}+H_{k} \hat{V}_{k}+\xi_{1}(k, \hat{\epsilon}) W_{-1}+\xi_{2}(k, \hat{\epsilon}) W_{1}\right. \\
\left.\quad+\left(\Omega_{0 k}+\rho_{0 k} H_{k}\right) \eta_{t} \xi_{3}(k, \epsilon, \hat{\epsilon})+W_{k}\right] H_{k}^{*}
\end{aligned}
$$

where

$$
\begin{aligned}
\hat{U}_{k}= & \sum_{m \in \mathcal{I}, m \neq k} \Omega_{m k} X_{m} \psi(m-k, \epsilon-\hat{\epsilon}) \\
& +\sum_{m \in \mathcal{I}} \Omega_{m k} X_{m} \phi(m-k, \epsilon-\hat{\epsilon}) \\
\hat{V}_{k}= & \sum_{m \in \mathcal{I}, m \neq k} \rho_{m k} X_{m} \psi(m-k, \epsilon-\hat{\epsilon}) \\
& +\sum_{m \in \mathcal{I}} \rho_{m k} X_{m} \phi(m-k, \epsilon-\hat{\epsilon}) \\
\phi(m, k, \epsilon, \hat{\epsilon})= & \xi_{1}(k, \hat{\epsilon}) \psi(m+1, \epsilon-\hat{\epsilon}) \\
& +\xi_{2}(k, \hat{\epsilon}) \psi(m-1, \epsilon-\hat{\epsilon}) .
\end{aligned}
$$

When $|\mathcal{I}|$ is reasonably large, we can evoke the CLT to approximate the random variables $\hat{U}_{k}$ and $\hat{V}_{k}$ as zero-mean complex Gaussian random variables with variances given, respectively, in (47) and (48), shown at the bottom of the next page.

We can rewrite (43) as

$$
\widetilde{Y}_{k} H_{k}^{*}=\alpha\left|H_{k}\right|^{2} X_{k}+\hat{Z}_{k}
$$

where

$$
\begin{aligned}
\hat{Z}_{k}=\left[\hat{U}_{k}+H_{k} \hat{V}_{k}+\left(\Omega_{0 k}+\rho_{0 k} H_{k}\right) \eta_{t} \xi_{3}(k, \epsilon, \hat{\epsilon})\right. \\
\left.+\xi_{1}(k, \hat{\epsilon}) W_{-1}+\xi_{2}(k, \hat{\epsilon}) W_{1}+W_{k}\right] H_{k}^{*}
\end{aligned}
$$

$$
\Delta \eta_{r}=\frac{\psi^{*}(1,-\hat{\epsilon}) I_{-1}^{\mathrm{ICI}}+\psi^{*}(-1,-\hat{\epsilon}) I_{1}^{\mathrm{ICI}}+\zeta_{1}(\epsilon, \hat{\epsilon}) H_{0} \eta_{t}+\psi^{*}(1,-\hat{\epsilon}) W_{-1}+\psi^{*}(-1,-\hat{\epsilon}) W_{1}}{\sqrt{N}\left(|\psi(1,-\hat{\epsilon})|^{2}+|\psi(-1,-\hat{\epsilon})|^{2}\right)}
$$

$$
E\left[\left|\Delta \eta_{r}\right|^{2}\right]=\frac{\zeta_{2}(\epsilon, \hat{\epsilon})+\left|\eta_{t}\right|^{2} N\left|\zeta_{1}(\epsilon, \hat{\epsilon})\right|^{2}+\left(N|\psi(1,-\hat{\epsilon})|^{2}+N|\psi(-1,-\hat{\epsilon})|^{2}+1\right) N_{0}}{N^{2}\left[|\psi(1,-\hat{\epsilon})|^{2}+|\psi(-1,-\hat{\epsilon})|^{2}\right]^{2}}
$$

$$
\begin{aligned}
\zeta_{2}(\epsilon, \hat{\epsilon})=\frac{E_{s}}{N} \sum_{m \in \mathcal{I}}[\mid & \left.\psi^{*}(1,-\hat{\epsilon})\right|^{2}|\psi(m+1, \epsilon-\hat{\epsilon})|^{2}+\left|\psi^{*}(-1,-\hat{\epsilon})\right|^{2}|\psi(m-1, \epsilon-\hat{\epsilon})|^{2} \\
& \left.+2 \operatorname{Re}\left[\psi^{*}(1,-\hat{\epsilon}) \psi(-1,-\hat{\epsilon}) \psi_{1}(m+1, \epsilon-\hat{\epsilon}) \psi^{*}(m-1, \epsilon-\hat{\epsilon})\right]\right] /\left[|\psi(1,-\hat{\epsilon})|^{2}+|\psi(-1,-\hat{\epsilon})|^{2}\right]^{2}
\end{aligned}
$$


can be approximated as a Gaussian random variable with mean $\mu_{\hat{z}_{k}}$ and variance $\sigma_{\hat{z}_{k}}^{2}$ as

$$
\begin{aligned}
\mu_{\hat{Z}_{k}}= & \rho_{0 k} \eta_{t} \xi_{3}(k, \epsilon, \hat{\epsilon})\left|H_{k}\right|^{2} \\
\sigma_{\hat{Z}_{k}}^{2}= & {\left[\begin{array}{l}
\sigma_{\hat{U}_{k}}^{2}+\left|H_{k}\right|^{2} \sigma_{\hat{V}_{k}}^{2}+\left(1-\left|\rho_{0 k}\right|^{2}\right)\left|\eta_{t}\right|^{2}\left|\xi_{3}(k, \epsilon, \hat{\epsilon})\right|^{2} \\
\quad \\
\left.\quad+\left(\left|\xi_{1}(k, \hat{\epsilon})\right|^{2}+\left|\xi_{2}(k, \hat{\epsilon})\right|^{2}+1\right) N_{0}\right]\left|H_{k}\right|^{2}
\end{array}\right.}
\end{aligned}
$$

Comparing $\mu_{\hat{z}_{k}}$ and $\sigma_{\hat{z}_{k}}^{2}$ to $\mu_{z_{k}}$ and $\sigma_{z_{k}}^{2}$, we know that the dc offset canceller eliminates the effect of $\eta_{r}$ on the mean $\mu_{\hat{z}_{k}}$ at the expense of increasing the noise variance $\sigma_{\hat{z}_{k}}^{2}$. Following the same procedures given in the previous section, we can derive the conditional SERs for the BPSK, QPSK, and 16-QAM OFDM signals after dc offset cancellation by replacing the variables $\mu_{z_{k}}$ and $\sigma_{z_{k}}^{2}$ by $\mu_{\hat{z}_{k}}$ and $\sigma_{\hat{z}_{k}}^{2}$ in the corresponding SER formulas (23), (54), and (66), respectively.

\section{NumericAl Results}

We consider an OFDM system with $N=64$ subcarriers The effective OFDM symbol period is $T=3.2 \mu \mathrm{s}$, and the subcarrier frequency spacing $f_{s}=1 / T$ is $312.5 \mathrm{kHz}$. The maximum allowable CFO is $250 \mathrm{kHz}$, and the corresponding normalized CFO $\epsilon$ is 0.8 . The power delay profile of the multipath Rayleigh fading channel is exponentially decaying, and the root mean square (RMS) delay spread is equal to $100 \mathrm{~ns}$. We assume that the channel is fixed for the whole frame and independently changes from frame to frame. These parameters and assumptions are typical for indoor WLAN applications.

Figs. 2-4 show the average SER performance versus symbol SNR $E_{s} / N_{0}$ for BPSK-, QPSK-, and 16-QAM-modulated OFDM signals in multipath Rayleigh fading channels, where the normalized CFO $\epsilon$ is 0.4 , and the magnitude of the dc offset $\eta_{r}$ ranges from 0 to $0.08 \sqrt{E_{s}}$. All subcarriers except the dc subcarrier are used for data transmission. In all three figures, the solid lines are obtained from computer simulations, and the markers are computed from theoretical analyses. It can be seen that the theoretical results very well coincide with the simulation results. As the dc offset $\left|\eta_{r}\right|$ increases, the SER degradation becomes more severe, and an error floor appears. This implies that the effect of dc offset dominates the SER performance in the high SNR region. Moreover, since the constellation points of 16-QAM modulation is more crowded than those of BPSK and QPSK modulations, 16-QAM modulation is more sensitive to the effect of dc offset. For a higher order modulation like

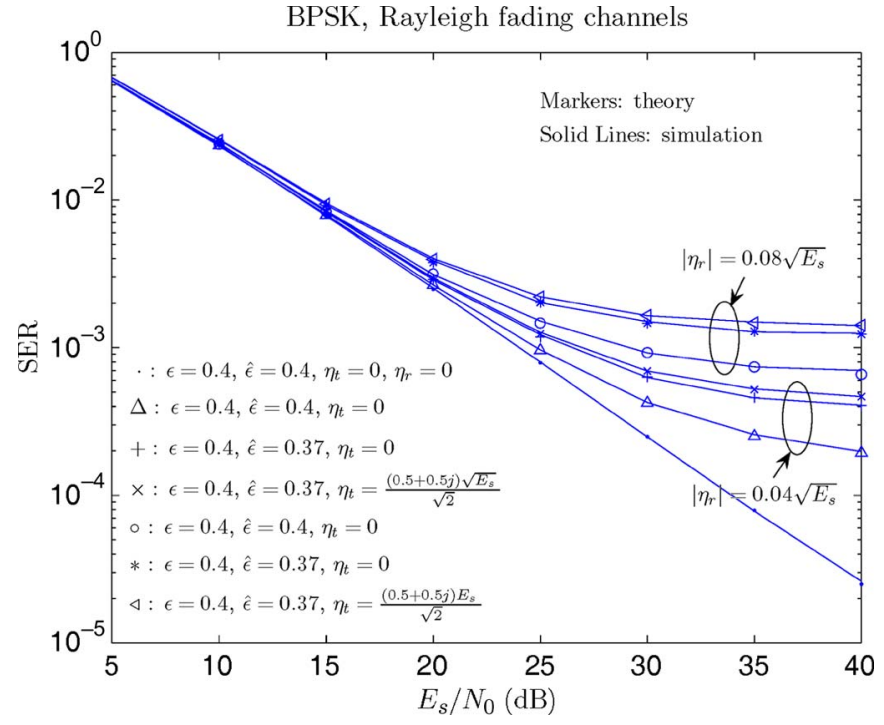

Fig. 2. Effects of dc offset on the SER of BPSK-modulated OFDM signals in multipath Rayleigh fading channels. $\epsilon=0.4$.

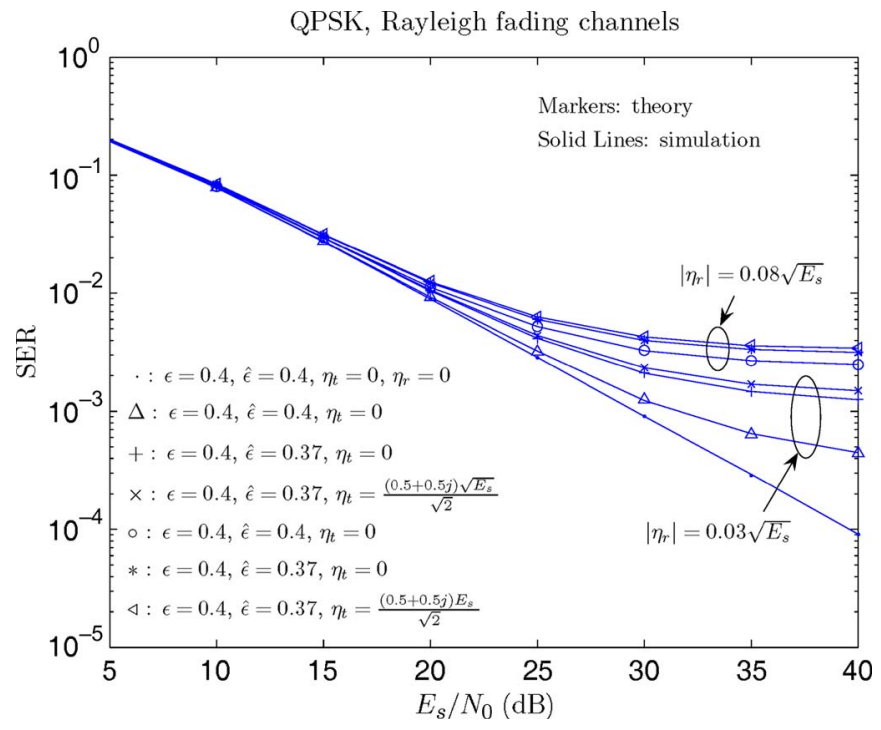

Fig. 3. Effects of dc offset on the SER of QPSK-modulated OFDM signals in multipath Rayleigh fading channels. $\epsilon=0.4$.

64-QAM, it is expected that the dc offset more seriously aggravates the performance degradation. In practical OFDM systems, the dc offset power should be less than the subcarrier power by at least $30 \mathrm{~dB}$ to avoid performance degradation, particularly when high-order modulation is employed.

$$
\begin{aligned}
\sigma_{\hat{U}_{k}}^{2}= & \sum_{m \in \mathcal{I}}\left(1-\left|\rho_{m k}\right|^{2}\right) E_{s}|\phi(m, k, \epsilon, \hat{\epsilon})|^{2} \\
& +\sum_{m \in \mathcal{I}, m \neq k}\left(1-\left|\rho_{m k}\right|^{2}\right) E_{s}\left(|\psi(m-k, \epsilon-\hat{\epsilon})|^{2}+2 \operatorname{Re}\left[\psi^{*}(m-k, \epsilon-\hat{\epsilon}) \phi(m, k, \epsilon, \hat{\epsilon})\right]\right) \\
\sigma_{\hat{V}_{k}}^{2}= & \sum_{m \in \mathcal{I}}\left|\rho_{m k}\right|^{2} E_{s}|\phi(m, k, \epsilon, \hat{\epsilon})|^{2} \\
& +\sum_{m \in \mathcal{I}, m \neq k}\left|\rho_{m k}\right|^{2} E_{s}\left(|\psi(m-k, \epsilon-\hat{\epsilon})|^{2}+2 \operatorname{Re}\left[\psi^{*}(m-k, \epsilon-\hat{\epsilon}) \phi(m, k, \epsilon, \hat{\epsilon})\right]\right)
\end{aligned}
$$




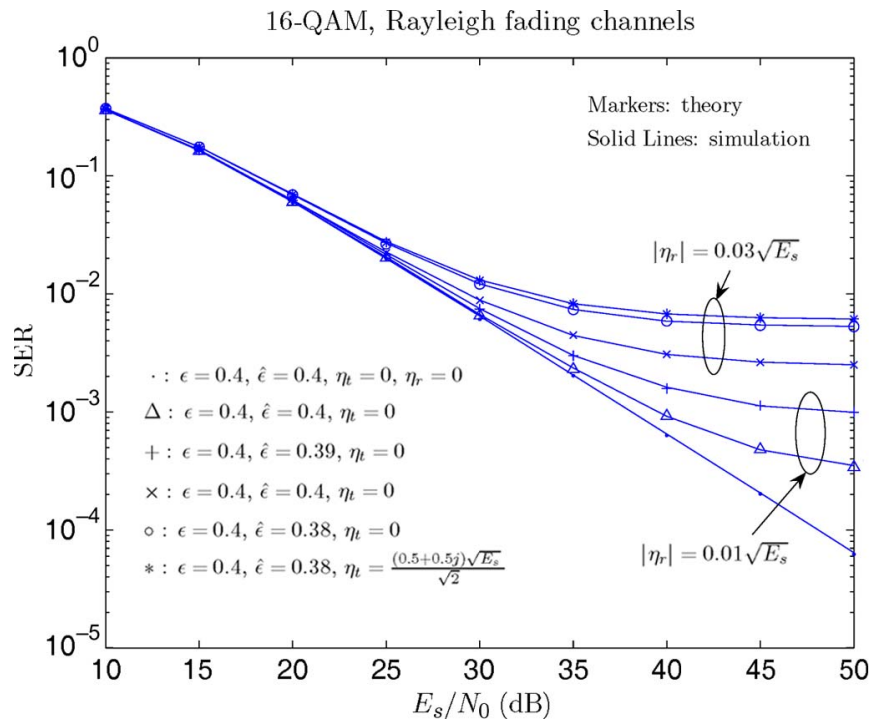

Fig. 4. Effects of dc offset on the SER of 16-QAM-modulated OFDM signals in multipath Rayleigh fading channels. $\epsilon=0.4 .\left|\eta_{r}\right|=0.01 \sqrt{E_{s}}$, and $0.03 \sqrt{E_{s}}$.

In addition to the performance loss caused by the receiver dc offset $\eta_{r}$, we also show the effects of imperfect CFO compensation and transmitter dc offset $\eta_{t}$ on SER in Figs. 2-4. When the estimated CFO $\hat{\epsilon}$ is not equal to the true CFO $\epsilon$, the resulting ICI and the dc offset interference from $\eta_{t}$ further degrade the system performance. To illustrate the influence of transmitter dc offset $\eta_{t}$ on system SER performance, we consider a very large $\eta_{t}$ equal to $(0.5+0.5 j) \sqrt{E_{s} / 2}$. Since the mismatch CFO $\epsilon-\hat{\epsilon}$ is small in practical OFDM systems, the amount of interference caused by $\eta_{t}$ is quite small, and even $\eta_{t}$ is unrealistically large as $(0.5+0.5 j) \sqrt{E_{s} / 2}$. In this case, the overall interference is dominated by the ICI due to CFO mismatch. This fact justifies the design of our dc cancellation scheme, where only the receiver dc offset $\eta_{r}$ is estimated and compensated.

In addition to the average SER, it is interesting to understand the effect of dc offset on the SER of each subcarrier. Fig. 5 shows the curves of SERs versus subcarrier index for the 16-QAM-modulated OFDM signal in multipath Rayleigh fading channels. The symbol SNR $E_{s} / N_{0}$ is $40 \mathrm{~dB}$, which results in SER of around $10^{-3}$ for the perfect synchronization case (i.e., no CFO and dc offset). For coded OFDM systems, the operating SNR is much lower due to the large coding gains. The transmitter dc offset $\eta_{t}$ is set to 0 , and the SER of the dc subcarrier is not shown in the figure because we assume no data symbol is transmitted at the dc subcarrier. When the receiver dc offset $\eta_{r}$ is zero, all subcarriers have the same SER of $6.3 \times 10^{-4}$. As the magnitude of receiver dc offset $\left|\eta_{r}\right|$ increases, the SER of each subcarrier becomes a decreasing function of the subcarrier index magnitude $|k|$. The performance degradation is more severe for those subcarriers surrounding the dc subcarrier. When CFO estimation is not perfect, the ICI dominates the total interference and boosts the SER to around $3.8 \times 10^{-3}$ when the magnitude of subcarrier index $|k|$ is greater than 20 .

The effects of CFO in the presence of dc offset on the SER performance for 16-QAM-modulated OFDM signals are shown

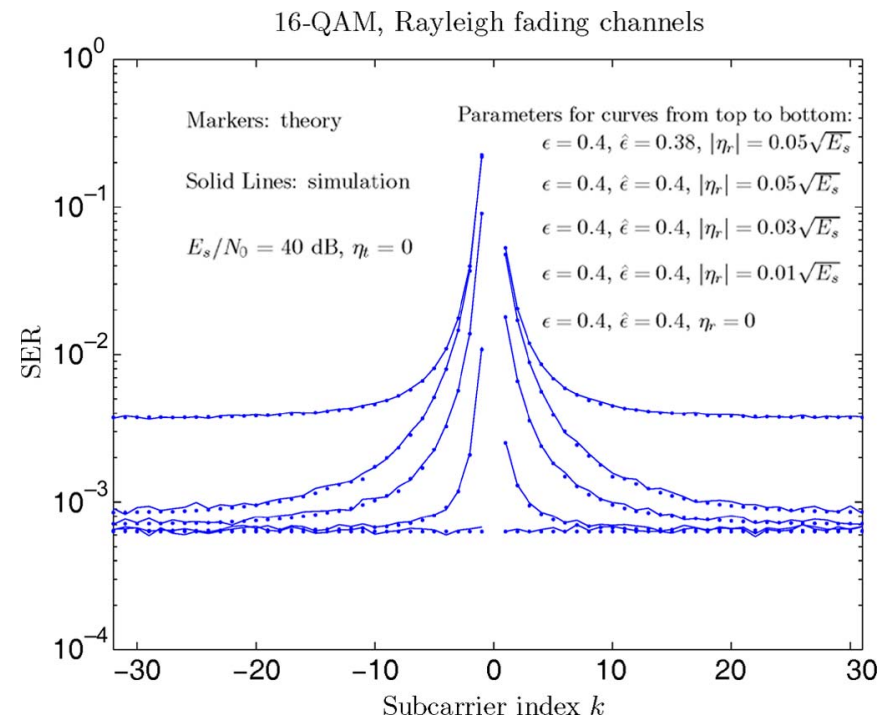

Fig. 5. Effects of CFO on the subcarrier SER of 16-QAM-modulated OFDM signals in multipath Rayleigh fading channels. $\epsilon=0.4 . E_{s} / N_{0}=40 \mathrm{~dB}$.

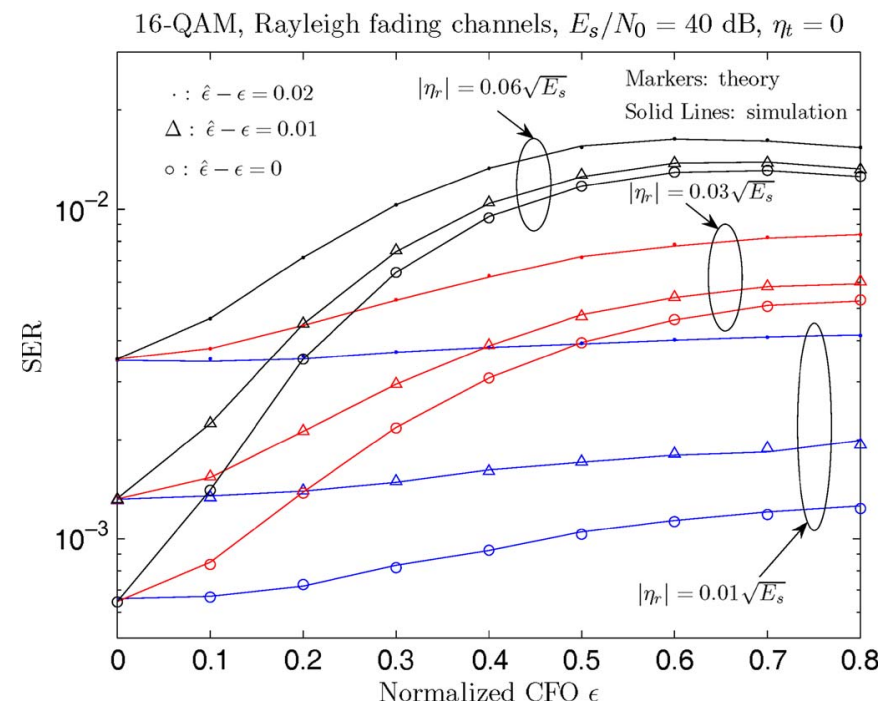

Fig. 6. Effects of CFO on the SER of 16-QAM-modulated OFDM signals in the presence of dc offsets $\left|\eta_{r}\right|=0.01 \sqrt{E_{s}}, 0.03 \sqrt{E_{s}}$, and $0.06 \sqrt{E_{s}}$. $E_{s} / N_{0}=40 \mathrm{~dB} \cdot \eta_{t}=0$.

in Fig. 6. The SNR is $40 \mathrm{~dB}$, and the transmitter dc offset $\eta_{t}$ is 0 . We consider three receiver dc offsets $\eta_{r}=0.01 \sqrt{E_{s}}$, $0.03 \sqrt{E_{s}}$, and $0.06 \sqrt{E_{s}}$ and vary the normalized $\mathrm{CFO} \epsilon$ from 0 to its maximum 0.8 . The effects of imperfect CFO estimation are also included in the figure. When CFO compensation is perfect, it can be seen that the influence of normalized CFO $\epsilon$ on the dc offset through (10) becomes more apparent as $\epsilon$ increases from 0 to 0.8 . The reason behind this phenomenon is that the CFO compensation spreads the dc offset energy and causes interference to all other subcarriers. Since the system does not transmit data at the dc subcarrier, more dc energy spread means more interference to other subcarriers and, hence, more performance degradation.

The SER performance of the proposed dc offset cancellation scheme for 16-QAM-modulated OFDM signals is shown in Fig. 7, where we set $\epsilon=0.4$ and $\left|\eta_{r}\right|=0.04 \sqrt{E_{s}}$. When the 


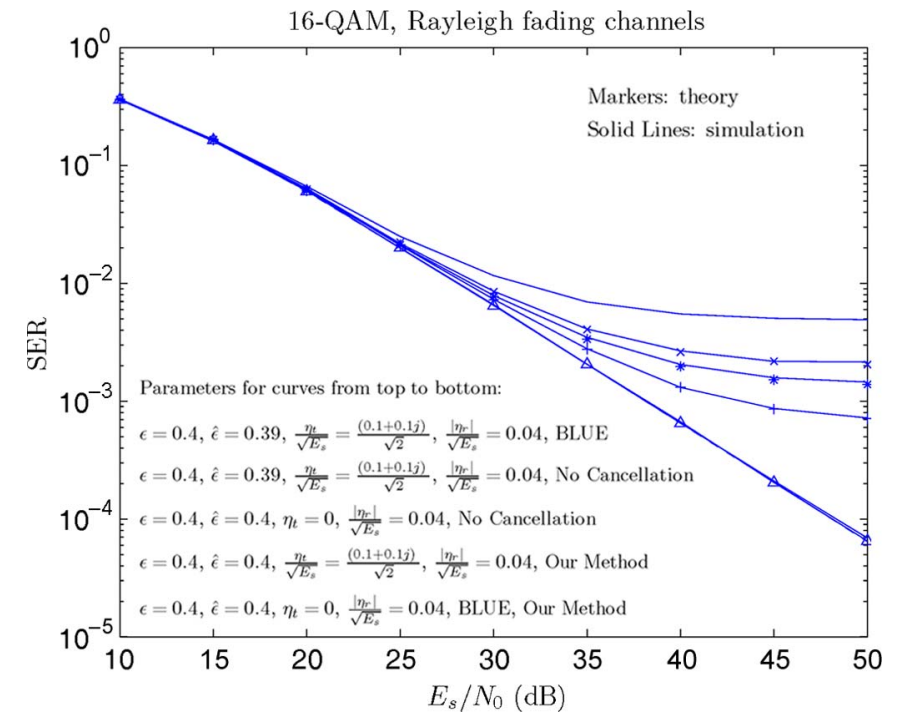

Fig. 7. SER performance of dc offset cancellor for 16-QAM-modulated OFDM signals in multipath Rayleigh fading channels.

transmitter dc offset $\eta_{t}$ is zero and the CFO is perfectly corrected (i.e., $\hat{\epsilon}=\epsilon=0.4$ ), it can be seen that both the proposed $\mathrm{dc}$ offset cancellation scheme and the BLUE of dc offset proposed in [14] can effectively compensate the receiver dc offset $\eta_{r}$. The analytical results and the simulation results are well matched. When the transmitter dc offset $\eta_{t}$ is equal to $(0.1+0.1 j) \sqrt{E_{s}} / \sqrt{2}$ and the estimated CFO $\hat{\epsilon}$ is equal to 0.39 , our proposed method can still effectively estimate and correct the dc offset while the SER performance of BLUE significantly degrades. This result is not surprising because the BLUE of dc offset is derived under the consideration of receiver dc offset only, and its performance is not robust when the transmitter dc offset $\eta_{t}$ is present.

Fig. 8 illustrates the performance improvement of dc offset cancellation on the subcarrier SER for 16-QAM-modulated OFDM signals. The parameters are the same as those used in Fig. 7. With the proposed dc offset cancellation scheme, the performance losses at the subcarriers around the dc subcarrier are greatly mitigated. Moreover, the good agreement between the analysis and simulation results further validates the correctness of our analysis.

The SER performance versus the normalized CFO $\epsilon$ for 16-QAM-modulated OFDM signals is shown in Fig. 9, where $E_{s} / N_{0}$ is fixed at $40 \mathrm{~dB}$. The transmitter and receiver dc offsets are $\eta_{t}=(0.1+0.1 j) \sqrt{E_{s}} / \sqrt{2}$ and $\left|\eta_{r}\right|=0.03 \sqrt{E_{s}}$. To illustrate the effect of imperfect CFO compensation, we consider three cases, i.e., $\hat{\epsilon}-\epsilon=0,0.01$, and 0.02. Without dc offset cancellation, the SER curves depend on the CFO $\epsilon$, which controls the spread of dc offset energy. After the dc offset cancellation, the SER performance is dominated by the ICI and is almost insensitive to the variation of the normalized CFO $\epsilon$.

Finally, it is worth noting that we do not consider any specific $\mathrm{CFO}$ estimator in this paper for the simplicity of analysis. When dc offsets are present, the CFO estimator must take dc offsets into account to have high accuracy. With proper design, the CFO estimator can achieve satisfactory performance at low complexity, irrespective of the dc offset presence [21].

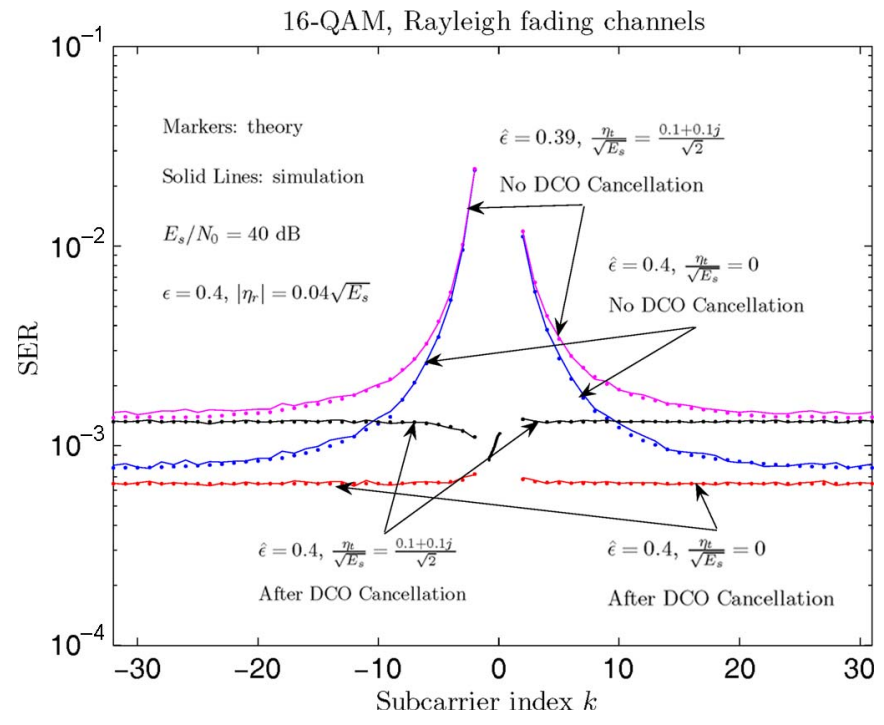

Fig. 8. Subcarrier SER of 16-QAM-modulated OFDM signals with/without dc offset cancellation in multipath Rayleigh fading channels.

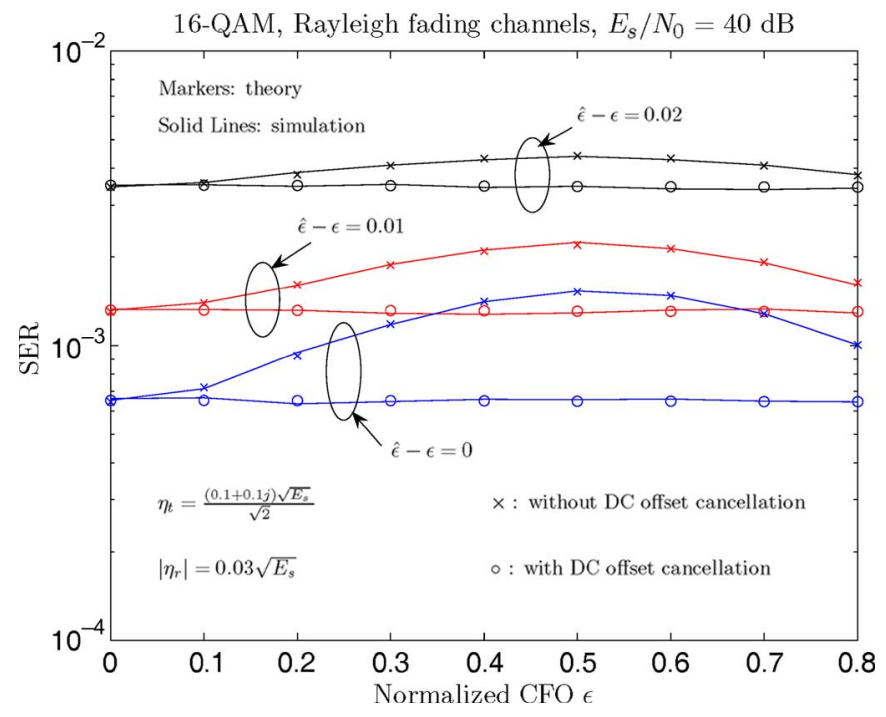

Fig. 9. Effects of CFO on the SER of 16-QAM-modulated OFDM signals in the presence of dc offsets $\eta_{t}=(0.1+0.1 j) \sqrt{E_{s}} / \sqrt{2}$ and $\left|\eta_{r}\right|=0.03 \sqrt{E_{s}}$. $E_{s} / N_{0}=40 \mathrm{~dB}$.

\section{CONCLUSION}

We have studied the effects of dc offset on the SER performance of OFDM systems with BPSK, QPSK, and 16-QAM modulations in multipath Rayleigh fading channels. The transmitter and receiver dc offsets were modeled and analyzed in this paper. Under the general setting of imperfect CFO compensation, we derived the analytical SER formulas characterizing the relationships among the SER, CFO, and dc offsets. When the compensation of dc offset is necessary, we proposed and analyzed a low-complexity dc offset cancellation scheme that has superior performance over wide ranges of the CFO and dc offsets. In practical situations, the $\mathrm{CFO}$ and dc offset can jointly be estimated to achieve better performance. The BER analysis for OFDM systems with the estimated CFO and dc offset will be left for future study. 


\section{APPENDIX}

BER ANALYSIS OF QPSK AND

16-QAM CONSTELLATIONS

\section{A. Quadrature Phase Shift Keying}

The four constellation points of QPSK modulation are

$$
\begin{aligned}
C_{0}=\frac{(1+j) \sqrt{E_{s}}}{\sqrt{2}}, & C_{1}=\frac{(-1+j) \sqrt{E_{s}}}{\sqrt{2}} \\
C_{2}=\frac{(-1-j) \sqrt{E_{s}}}{\sqrt{2}}, & C_{3}=\frac{(1-j) \sqrt{E_{s}}}{\sqrt{2}}
\end{aligned}
$$

where $j=\sqrt{-1}$, and $E_{s}$ is the average symbol energy. Assuming perfect CSI is available at the receiver, it is obvious that the decision boundaries are the real and imaginary axes. Therefore, the conditional SER of the symbol transmitted at subcarrier $k \in \mathcal{I}$ is given by

$$
P_{s}^{\mathrm{QPSK}}\left(k \mid H_{k}\right)=1-\frac{1}{4}\left(P_{k, 0}+P_{k, 1}+P_{k, 2}+P_{k, 3}\right)
$$

where

$$
\begin{aligned}
P_{k, 0}= & \operatorname{Pr}\left(\operatorname{Re}\left[Y_{k} H_{k}^{*}\right]>0 \mid X_{k}=C_{0}, H_{k}\right) \\
& \times \operatorname{Pr}\left(\operatorname{Im}\left[Y_{k} H_{k}^{*}\right]>0 \mid X_{k}=C_{0}, H_{k}\right) \\
P_{k, 1}= & \operatorname{Pr}\left(\operatorname{Re}\left[Y_{k} H_{k}^{*}\right]<0 \mid X_{k}=C_{1}, H_{k}\right) \\
& \times \operatorname{Pr}\left(\operatorname{Im}\left[Y_{k} H_{k}^{*}\right]>0 \mid X_{k}=C_{1}, H_{k}\right) \\
P_{k, 2}= & \operatorname{Pr}\left(\operatorname{Re}\left[Y_{k} H_{k}^{*}\right]<0 \mid X_{k}=C_{2}, H_{k}\right) \\
& \times \operatorname{Pr}\left(\operatorname{Im}\left[Y_{k} H_{k}^{*}\right]<0 \mid X_{k}=C_{2}, H_{k}\right) \\
P_{k, 3}= & \operatorname{Pr}\left(\operatorname{Re}\left[Y_{k} H_{k}^{*}\right]>0 \mid X_{k}=C_{3}, H_{k}\right) \\
& \times \operatorname{Pr}\left(\operatorname{Im}\left[Y_{k} H_{k}^{*}\right]<0 \mid X_{k}=C_{3}, H_{k}\right)
\end{aligned}
$$

are the correct probabilities of the constellation points $C_{0}, C_{1}$, $C_{2}$, and $C_{3}$, respectively. The notation $\operatorname{Im}[x]$ represents the imaginary part of the complex number $x$.

The probabilities $\operatorname{Pr}\left(\operatorname{Re}\left[Y_{k} H_{k}^{*}\right]<0 \mid X_{k}, H_{k}\right) \quad$ and $\operatorname{Pr}\left(\operatorname{Re}\left[Y_{k} H_{k}^{*}\right]>0 \mid X_{k}, H_{k}\right)$ given in (55)-(58) can explicitly be expressed as

$$
\begin{aligned}
\operatorname{Pr} & \left(\operatorname{Re}\left[Y_{k} H_{k}^{*}\right]<0 \mid X_{k}, H_{k}\right) \\
& =Q\left(\frac{\operatorname{Re}\left[\alpha\left|H_{k}\right|^{2} X_{k}+\mu_{z_{k}}\right]}{\sqrt{\sigma_{z_{k}}^{2} / 2}}\right) \\
\operatorname{Pr} & \left(\operatorname{Re}\left[Y_{k} H_{k}^{*}\right]>0 \mid X_{k}, H_{k}\right) \\
& =1-Q\left(\frac{\operatorname{Re}\left[\alpha\left|H_{k}\right|^{2} X_{k}+\mu_{z_{k}}\right]}{\sqrt{\sigma_{z_{k}}^{2} / 2}}\right) .
\end{aligned}
$$

Similarly, the probabilities $\operatorname{Pr}\left(\operatorname{Im}\left[Y_{k} H_{k}^{*}\right]<0 \mid X_{k}, H_{k}\right)$ and $\operatorname{Pr}\left(\operatorname{Im}\left[Y_{k} H_{k}^{*}\right]>0 \mid X_{k}, H_{k}\right)$ can be obtained by replacing $\operatorname{Re}[\cdot]$ in (59) and (60) by $\operatorname{Im}[\cdot]$, respectively. Finally, the average SER of OFDM signals using QPSK modulation is

$$
P_{s}^{\mathrm{QPSK}}=\frac{1}{|\mathcal{I}|} \sum_{k \in \mathcal{I}} \mathbb{E}_{H_{k}}\left[P_{s}^{\mathrm{QPSK}}\left(k \mid H_{k}\right)\right] .
$$

Similar to the BPSK case, this expectation can be evaluated by numerical integration.

\section{B. 16-QAM}

We denote the sixteen constellation points of 16-QAM by

$$
C_{4 q+i}=[(2 i-3)+j(2 q-3)] d, \quad i, q=0,1,2,3
$$

where $d=\sqrt{E_{s} / 10}$. For subcarrier $k$, the conditional probability of correct detection of constellation point $C_{i}$ under the perfect CSI assumption is

$$
\begin{gathered}
P_{k, c}\left(i \mid H_{k}\right)=\operatorname{Pr}\left(d_{i}^{l_{1}}<\frac{\operatorname{Re}\left[Y_{k} H_{k}^{*}\right]}{\left|H_{k}\right|^{2}}<d_{i}^{u_{1}} \mid X_{k}=C_{i}, H_{k}\right) \\
\times \operatorname{Pr}\left(d_{i}^{l_{2}}<\frac{\operatorname{Im}\left[Y_{k} H_{k}^{*}\right]}{\left|H_{k}\right|^{2}}<d_{i}^{u_{2}} \mid X_{k}=C_{i}, H_{k}\right)
\end{gathered}
$$

where the upper and lower limits $d_{i}^{l_{1}}, d_{i}^{u_{1}}, d_{i}^{l_{2}}, d_{i}^{u_{2}}$ are taken from the set $\mathcal{S}=\{-\infty,-2 d, 0,2 d, \infty\}$. Then, $\operatorname{Pr}\left(d_{i}^{l_{1}}<\right.$ $\left.\left(\operatorname{Re}\left[Y_{k} H_{k}^{*}\right] /\left|H_{k}\right|^{2}\right)<d_{i}^{u_{1}} \mid X_{k}=C_{i}, H_{k}\right) \quad$ and $\operatorname{Pr}\left(d_{i}^{l_{2}}<\right.$ $\left.\left(\operatorname{Im}\left[Y_{k} H_{k}^{*}\right] /\left|H_{k}\right|^{2}\right)<d_{i}^{u_{2}} \mid X_{k}=C_{i}, H_{k}\right) \quad$ can explicitly be computed by

$$
\begin{gathered}
\operatorname{Pr}\left(d_{i}^{l_{1}}<\frac{\operatorname{Re}\left[Y_{k} H_{k}^{*}\right]}{\left|H_{k}\right|^{2}}<d_{i}^{u_{1}} \mid X_{k}=C_{i}, H_{k}\right) \\
=Q\left(\frac{d_{i}^{l_{1}}\left|H_{k}\right|^{2}-\operatorname{Re}\left[\alpha\left|H_{k}\right|^{2} X_{k}+\mu_{z_{k}}\right]}{\sqrt{\sigma_{z_{k}}^{2} / 2}}\right) \\
-Q\left(\frac{d_{i}^{u_{1}}\left|H_{k}\right|^{2}-\operatorname{Re}\left[\alpha\left|H_{k}\right|^{2} X_{k}+\mu_{z_{k}}\right]}{\sqrt{\sigma_{z_{k}}^{2} / 2}}\right) \\
\operatorname{Pr}\left(d_{i}^{l_{2}}<\frac{\operatorname{Im}\left[Y_{k} H_{k}^{*}\right]}{\left|H_{k}\right|^{2}}<d_{i}^{u_{2}} \mid X_{k}=C_{i}, H_{k}\right) \\
=Q\left(\frac{d_{i}^{l_{2}}\left|H_{k}\right|^{2}-\operatorname{Im}\left[\alpha\left|H_{k}\right|^{2} X_{k}+\mu_{z_{k}}\right]}{\sqrt{\sigma_{z_{k}}^{2} / 2}}\right) \\
-Q\left(\frac{d_{i}^{u_{2}}\left|H_{k}\right|^{2}-\operatorname{Im}\left[\alpha\left|H_{k}\right|^{2} X_{k}+\mu_{z_{k}}\right]}{\sqrt{\sigma_{z_{k}}^{2} / 2}}\right) .
\end{gathered}
$$

For subcarrier $k$, the average conditional SER of 16-QAMmodulated OFDM signals is

$$
P_{s}^{16-\mathrm{QAM}}\left(k \mid H_{k}\right)=1-\frac{1}{16} \sum_{i=0}^{15} P_{k, c}\left(i \mid H_{k}\right) .
$$

Finally, the conditional SER $P_{s}^{16-\mathrm{QAM}}(k)$ is averaged over the channel frequency responses $H_{k}$ and all subcarriers in the active set $\mathcal{I}$ to obtain the final SER

$$
P_{s}^{16-\mathrm{QAM}}=\frac{1}{|\mathcal{I}|} \sum_{k \in \mathcal{I}} \mathbb{E}_{H_{k}}\left[P_{s}^{16-\mathrm{QAM}}\left(k \mid H_{k}\right)\right] .
$$




\section{ACKNOWLEDGMENT}

The author would like to thank the associate editor and the anonymous reviewers, whose comments have improved the quality and presentation of this paper.

\section{REFERENCES}

[1] B. Razavi, RF Microelectronics. Englewood Cliffs, NJ: Prentice-Hall, 1998.

[2] S.-B. Park, "Compact high performance analog CMOS baseband design solutions for multistandard wireless transceivers," Ph.D. dissertation, Ohio State Univ., Columbus, OH, 2006.

[3] R. Svitek and S. Raman, "DC offsets in direct-conversion receivers: Characterization and implications," IEEE Microw. Mag., vol. 6, no. 3, pp. 76-86, Sep. 2005.

[4] M. Gustavsson, J. J. Wikner, and N. N. Tan, CMOS Data Converters for Communications. Norwell, MA: Kluwer, 2000.

[5] J. K. Cavers and M. W. Liao, "Adaptive compensation for imbalance and offset losses in direct conversion transceivers," IEEE Trans. Veh. Technol., vol. 42, no. 4, pp. 581-588, Nov. 1993.

[6] B. Lindoff, "Using a direct conversion receiver in EDGE terminalsA new DC offset compensation algorithm," in Proc. IEEE Pers. Indoor Mobile Radio Commun., 2000, pp. 959-963.

[7] B. Lindoff and P. Malm, "BER performance analysis of a direct conversion receiver," IEEE Trans. Commun., vol. 50, no. 5, pp. 856-865, May 2002.

[8] K. Witrisal, "Impact of DC-offsets and carrier feed-through on correlation-based frequency synchronization for OFDM," in Proc. 6th Int. OFDM Workshop, Hamburg, Germany, 2001, pp. 15-1-15-5.

[9] P. H. Moose, "A technique for orthogonal frequency division multiplexing frequency offset correction," IEEE Trans. Commun., vol. 42, no. 10, pp. 2908-2914, Oct. 1994.

[10] C. K. Ho, S. Sun, and P. He, "Low complexity frequency offset estimation in the presence of DC offset," in Proc. IEEE Int. Conf. Commun., May 2003, pp. 2051-2055.

[11] W. Eberle, J. Tubbax, B. Come, S. Donnay, H. De Man, and G. Gielen, "OFDM-WLAN receivers performance improvement using digital communication techniques," in Proc. IEEE Radio Wireless Conf., Aug. 2002, pp. 111-114.

[12] O. V. Popov, "Dynamic DC offset impact on the 802.11a receiver performance," in Proc. IEEE Int. Conf. Circuits Syst. Commun., Jun. 2002, pp. 250-253.

[13] H. Lin, T. Nakao, W. Lu, and K. Yamashita, "Subspace-based OFDM carrier frequency offset estimation in the presence of DC offset," in Proc. IEEE Int. Conf. Commun., Jun. 2007, pp. 2883-2887.

[14] S. Marsili, "DC offset estimation in OFDM based WLAN," in Proc. IEEE Global Commun. Conf., Dec. 2004, pp. 3531-3535.
[15] T. Maeda, H. Yano, S. Hori, N. Matsuno, T. Yamase, T. Tokairin, R. Walkington, N. Yoshida, K. Numata, K. Yanagisawa, Y. Takahashi, M. Fujii, and H. Hida, "Low-power-consumption direct-conversion CMOS transceiver for multi-standard 5-GHz wireless LAN systems with channel bandwidths of 5-20 MHz," IEEE J. Solid-State Circuits, vol. 41, no. 2, pp. 375-383, Feb. 2006.

[16] A. Behzad, K. A. Carter, H.-M. Chien, S. Wu, M.-A. Pan, C. P. Lee, Q. Li, J. C. Leete, S. Au, M. S. Kappes, Z. Zhou, D. Ojo, L. Zhang, A. Zolfaghari, J. Castanada, H. Darabi, B. Yeung, A. Rofougaran, M. Rofougaran, J. Trachewsky, T. Moorti, R. Gaikwad, A. Bagchi, J. S. Hammerschmidt, J. Pattin, J. J. Rael, and B. Marholev, "A fully integrated MIMO multiband direct conversion CMOS transceiver for WLAN applications (802.11n)," IEEE J. Solid-State Circuits, vol. 42, no. 12, pp. 2795-2808, Dec. 2007.

[17] L. Rugini and P. Banelli, "BER of OFDM systems impaired by carrier frequency offset in multipath fading channels," IEEE Trans. Wireless Commun., vol. 4, no. 5, pp. 2279-2288, Sep. 2005

[18] C.-H. Yih, "BER analysis of OFDM systems impaired by DC offset and carrier frequency offset in multipath fading channels," IEEE Commun. Lett., vol. 11, no. 11, pp. 842-844, Nov. 2007.

[19] Wireless LAN Media Access Control (MAC) and Physical Layer (PHY) Specification: High-Speed Physical Layer in the $5 \mathrm{GHz}$ Band, IEEE Std. 802.11a, Sep. 1999.

[20] Digital Video Broadcasting (DVB): Framing Structure, Channel Coding and Modulation for Terrestrial Television, EN 300744 V1.5.1, Nov. 2004.

[21] H. Lin, X. Wang, and K. Yamashita, "A low-complexity carrier frequency offset estimator independent of DC offset," IEEE Commun. Lett., vol. 12, no. 7 , pp. 520-522, Jul. 2008.

[22] M. Abramowitz and I. A. Stegun, Handbook of Mathematical Functions With Formulas, Graphs, and Mathematical Tables, 9th ed. New York: Dover, 1970

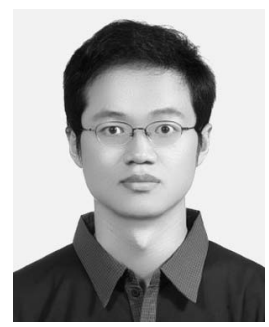

Chi-Hsiao Yih received the B.S. degree in control engineering from the National Chiao Tung University, Hsinchu, Taiwan, in 1993 and the M.S. and $\mathrm{Ph} . \mathrm{D}$. degrees in electrical engineering from the University of Maryland, College Park, in 1999 and 2003, respectively.

From 2003 to 2005, he was a Senior IC Design Engineer with Genesys Logic Inc. Since August 2005, he has been with the Department of Electrical Engineering, Tamkang University, Tamsui, Taiwan, where he is currently an Assistant Professor. His research interests include digital communications, error control coding, and statistical signal processing. 